\title{
COMPLETELY SEMINORMAL OPERATORS WITH BOUNDARY EIGENVALUES
}

BY

\section{KEVIN CLANCEY}

\begin{abstract}
For $f \in L^{2}(E)$ we consider the singular integral operator $T_{E} f(s)=s f(s)+\pi^{-1} \int_{E} f(t)(t-s)^{-1} d t$. These singular integral operators are a special case of operators acting on a Hilbert space with one dimensional self-commutator. We discover generalized eigenfunctions of the equation $T_{E} f=0$ and, for $p<2$, we will give an $L^{p}(E)$ solution of the equation $T_{E} f=x_{E}$. The main result of the paper is an example of a nonzero $L^{2}(E)$ solution of $T_{E} f=0$, with $\lambda=0$ a boundary point of the spectrum of $T_{E}$.
\end{abstract}

Let $R$ be the real line and $E$ a bounded Lebesgue measurable subset of $R$. For any $p, 1 \leq p \leq \infty$, the notation $L^{p}(E)$ will denote the usual Lebesgue space over $E$. Fix a function $\phi$ in $L^{\infty}(E)$. The operators of interest are defined for $f \in L^{p}(E), p>1$, by

$$
T_{\phi} f(s)=s f(s)+\pi^{-1} \phi(s) \int_{E}(t-s)^{-1} \bar{\phi}(t) f(t) d t .
$$

The singular integral is interpreted as a Cauchy principal value. The operators $T_{\phi}$ defined by (1) are bounded linear operators on $L^{p}(E)$, for $p>1$.

In the case where $p=2$ the operators defined by (1) are of special interest. Indeed, they are the prototype of an operator $T$ acting on a Hilbert space $H$, where $T^{*} T-T T^{*}$ is one dimensional (see, for example, Pincus [7] or Putnam [8]).

The spectrum of the operator $T_{\phi}$ defined by $(1)$ on $L^{2}(E)$ is described in [3] and Putnam [9]. Recently, the author, in [2], determined the essential spectrum of $T_{\phi}$ on $L^{2}(E)$. In this paper we initiate an attack on the problem of determining the eigenvalues of $T_{\phi}$. The only case where the point spectrum of $T_{\phi}$ has been computed is when $E$ is an interval and $\phi$ is a smooth function (see Putnam [9]).

We will be concerned mainly with the special case where the function $\phi$ in (1) is the characteristic function $\chi_{E}$ of $E$. In this case we will write $T_{E}$ for the operator $T_{\boldsymbol{x}_{E}}$. In $\oint_{3}$ we will determine explicitly some generalized solutions of

Presented to the Society, January 25, 1973 under the title Boundary eigenvalues of hyponormal operators; received by the editors May 9, 1972.

AMS (MOS) subject classifications (1970). Primary 47B35; Secondary $45 \mathrm{E} 05$.

Key words and phrases. Singular integral operator, seminormal operator. 
the equation $T_{E} f=\chi_{E}$. These solutions will permit us to write down some generalized eigenfunctions of the equation $T_{E} f=0$. In $\$ 4$, with severe restrictions on $E$, we will give some examples of $L^{2}(E)$ solutions of $T_{E} f=0$, where $\lambda=0$ is a boundary point of the spectrum of $T_{E}$ on $L^{2}(E)$.

The relation between the operators $T_{\phi}$ given by (1) on $L^{2}(E)$ and the theory of nonnormal operators on a Hilbert space has already been noted. More generally, an operator $T$ on a Hilbert space $H$ is called seminormal in case its self-commutator $T^{*} T-T T^{*}$ is semidefinite. Clearly, every operator $T$ for which $T^{*} T-T T^{*}$ is one dimensional is seminormal. A seminormal operator $T$ is said to be completely seminormal in case there are no nontrivial reducing subspaces for $T$ on which $T$ is a normal operator. An easy argument establishes that $T_{\phi}$ defined by (1) is completely seminormal if and only if $\phi(t) \neq 0$ a.e. on $E$.

An eigenvalue $\lambda$ of an operator $T$ on a Hilbert space $H$ is called a normal eigenvalue in case $(T-\lambda) x_{0}=0$ for $x_{0}$ in $H$ if and only if $\left(T^{*}-\bar{\lambda}\right) x_{0}=0$. It is obvious that a completely seminormal operator can have no normal eigenvalues. The following theorem of Putnam [10] and Stampfli [11] establishes that a boundary eigenvalue of a seminormal operator is often a normal eigenvalue.

Theorem. Suppose $\lambda_{0}$ is a boundary eigenvalue of a seminormal operator $T$. If $\lambda_{0}$ is accessible from the resolvent set of $T$, in the sense that there exists a sequence $\lambda_{n} \rightarrow \lambda_{0}$ satisfying $\left|\lambda_{n}-\lambda_{0}\right| / d_{T}\left(\lambda_{n}\right) \rightarrow 1$ (bere, $d_{T}(\lambda)$ denotes the distance from $\lambda$ to the spectrum), then $\lambda_{0}$ is a normal eigenvalue.

It is apparent from this theorem that boundary eigenvalues of completely seminormal operators are "hidden" in the boundary. This makes the construction of our examples in $\$ 4$ nontrivial.

Brennan [1] has given examples of seminormal (actually cosubnormal) operators with boundary eigenvalues. If $X$ is a compact set in the plane, let $R^{2}(X)$ denote the $L^{2}(d x d y)$ closure of the set of rational functions with poles off $X$. Brennan proves that if $X$ is nowhere dense, either $R^{2}(X)$ equals $L^{2}$ or the adjoint of multiplication by $z$ on $R^{2}(X)$ has a boundary eigenvalue. These examples do not have the property that the self-commutator is one dimensional.

It is hoped that this paper will lead to additional results on the fine structure of the spectrum of the operators defined by (1).

2. This section is concerned with preliminaries and develops some techniques for solving finite singular integral equations. The available literature on this problem (for measurable coefficients) is limited. Our main references are the book of Tricomi [12] and a paper of Widom [13].

If $f$ belongs to $L^{p}$, then we will denote the Hilbert transform of $f$ by $\tilde{f}(x)=$ $\pi^{-1} \int f(t)(t-x)^{-1} d t$; here, the singular integral is a Cauchy principal value. It is 
a well-known theorem of M. Riesz that, for $p>1, f \rightarrow \tilde{f}$ is a bounded linear operator on $L^{p}$. The Cauchy integral of a function $f \in L^{p}$, for $p>1$, is the function $C f$, which is separately holomorphic in the upper half-plane $\pi_{+}$and lower halfplane $\pi_{-}$, defined by $C f(z)=\pi^{-1} \int f(t)(t-z)^{-1} d t$. If $f \in L^{p}$, then the limits $C f^{+}(x)=\operatorname{Lim}_{y\rfloor 0} C f(x+i y)$ and $C f^{-}(x)=\operatorname{Lim}_{y ! 0} C f(x-i y)$ exist a.e. and satisfy the barrier relations

$$
C f^{+}-C f^{-}=2 i f, \quad C f^{+}+C f^{-}=2 \tilde{f} .
$$

The notation $H_{+}^{p}$ will be used for the Hardy space of the upper half-plane. To be more precise, for $p>0$, we define

$$
H_{+}^{p}=\left\{\Theta: \Theta \text { is analytic in } \pi_{+}, \sup _{y>0} \int|\Theta(x+i y)|^{p} d x<\infty\right\} .
$$

The usual Hardy space of the disc will be denoted by $H^{p}$.

The following theorem appears in Hille and Tamarkin [6].

Theorem 1. Let $p>1$ and $\Theta \in H_{+}^{p}$, then $\Theta^{+}(x)=\operatorname{Lim}_{y \downarrow 0} \Theta(x+i y)$ exists a.e. and belongs to $L^{p}$. The boundary function $\Theta^{+}$satisfies

$$
\operatorname{Re} \Theta^{+}=\left(\operatorname{Im} \Theta^{+}\right)^{\sim} \text {. }
$$

We next describe a technique for solving finite singular integral equations. Let $\theta$ be an essentially bounded real function with compact support. We define the function

$$
\Theta(z)=\exp \left[\pi^{-1} \int \theta(t)(t-z)^{-1} d t\right]-1, \quad \operatorname{Im} z \neq 0 .
$$

Clearly, the limit function $\Theta^{+}(x)=\operatorname{Lim}_{y ! 0} \Theta(x+i y)$ exists; in fact, using (1) we obtain

$$
\Theta^{+}=\exp [\tilde{\theta}+i \theta]-1
$$

When the function $\Theta$ defined by (4) is in $H_{+}^{p}$, for some $p>1$, then from Theorem 1

$$
\cos \theta \exp \tilde{\theta}-1=[(\sin \theta \exp \tilde{\theta})]^{2} \text {. }
$$

In $\$ 3$ we will show how some judicious choices of $\theta$ turn (6) into the identity $T_{E} f=\chi_{E}$.

Our first task is to obtain conditions sufficient for $\Theta$ defined by (4) to be in $H_{+}^{p}$, for some $p>1$. In fact we will prove, in Lemma 2 , that if $\Theta^{+}$given by (5) is in $L^{p}$, for some $p>1$, then $\Theta$ defined by (4) is in $H_{+}^{p}$. The reader is reminded that there exist functions analytic in $\pi_{+}$which have $L^{p}$ limit functions on the $x$-axis but fail to be in $H_{+}^{p}$. The function $\Theta(z)=e^{-i z} /(1-i z)$ behaves in this manner.

The next lemma is preparatory for Lemma 2. It is similar to a lemma of Gabriel [5]. First we introduce some notation. 
For each $y>0, \Gamma_{y}$ will denote the circular arc in the unit disc which is the image of the line segment $\{x+i y:-a \leq x \leq a\}$ under the map $\zeta=(i-z) /(i+z)$; here, $a>0$ is fixed.

Lemma 1. Suppose $f \in H^{p}$, for some $p>1$, then there exists for each $y_{0}>0$ a constant $M_{y_{0}}$ such that

$$
\sup _{0<y \leq y_{0}} \int_{\Gamma_{y}}|f(z)|^{p} d|z| \leq M_{y_{0}}
$$

Proof. If $f \in H^{p}$, then

$$
|f(\zeta)|^{p} \leq \int_{-\pi}^{\pi} \frac{1}{2 \pi} \operatorname{Re}\left[\frac{e^{i t}+\zeta}{e^{i t}-\zeta}\right]\left|f\left(e^{i t}\right)\right|^{p} d t .
$$

This result appears in Duren [4, p. 28]. Therefore,

$$
\int_{\Gamma_{y}}|f(\zeta)|^{p} d|\zeta| \leq \int_{-\pi}^{\pi}\left|f\left({ }_{2}{ }^{i t}\right)\right|^{p} \int_{\Gamma_{y}} \frac{1}{2 \pi} \operatorname{Re}\left[\frac{e^{i t}+\zeta}{e^{i t}-\zeta}\right] d|\zeta| d t .
$$

Clearly,

$$
\int_{\Gamma_{y}} \frac{1}{2 \pi} \operatorname{Re}\left[\frac{e^{i t}+\zeta}{e^{i t}-\zeta}\right] d|\zeta| \leq \int_{C_{y}} \frac{1}{2 \pi} \operatorname{Re}\left[\frac{e^{i t}+\zeta}{e^{i t}-\zeta}\right] d|\zeta|, \quad e^{i t} \neq-1
$$

where $C_{y}$ is the circle that is the image of $\operatorname{Im} z=y$ under $\zeta=(i-z) /(i+z)$. If $a_{y}$ is the center of $C_{y}$ (obviously, for $\left.y>0,-1<a_{y}<0\right)$ and if $r_{y}$ is.the radius of $C_{y}$, then when $e^{i t} \neq-1$, the mean value theorem for the harmonic function $\operatorname{Re}\left[\left(e^{i t}+\zeta\right) /\left(e^{i t}-\zeta\right)\right]$ permits one to conclude

$$
\int_{C_{y}} \frac{1}{2 \pi} \operatorname{Re}\left[\frac{e^{i t}+\zeta}{e^{i t}-\zeta}\right] d|\zeta| \leq \frac{2 r y}{1-\left|a_{y}\right|}
$$

It follows that

$$
\sup _{0<y \leq y 0} \int_{\Gamma_{y}}|f(\zeta)|^{p} d|\zeta| \leq \frac{2 \int_{-\pi}^{\pi}\left|f\left(e^{i t}\right)\right|^{p} d t}{1-\left|a_{y_{0}}\right|}
$$

This completes the proof of the lemma.

Lemma 2. Let $\theta$ be real valued, essentially bounded and bave compact support. Define $\Theta(z)$, for $\operatorname{Im} z>0$, by (4). If $\Theta^{+}$is in $L^{p}$, for some $p>1$, then $\Theta$ belongs to $H_{+}^{p}$.

Proof. Choose $a>b>1$ such that $\theta \equiv 0$ off $[-b, b]$. The presence of the factor -1 in the definition of $\Theta$ permits an easy power series expansion to prove that for every $p>1$ and $y_{0}>0$, there exists an $M_{p}$ such that

$$
\sup _{y>0} \int_{R \sim(-a, a)}|\Theta(x+i y)|^{p} d x<M_{p}
$$


and

$$
\sup _{y \geq y_{0}} \int_{-a}^{a}|\Theta(x+i y)|^{p} d x<M_{p} .
$$

For $\operatorname{Im} z \neq 0$, we define

$$
\Theta_{0}(z)=\exp \frac{1}{\pi} \int \frac{\theta(t)}{t-z} \frac{1+t z}{1+t^{2}} d t
$$

We have introduced the function $\Theta_{0}$ because it transfers over to a function on the unit disk in a simpler manner than does the function $\Theta$. From the observation

$$
\frac{1+t z}{\left(1+t^{2}\right)(t-z)}=\frac{1}{t-z}-\frac{t}{t^{2}+1}
$$

it is clear that $\Theta+1$ and $\Theta_{0}$ differ only by a multiplicative constant. Hence, there is an $N_{p}$ such that

$$
\sup _{0<y \leq y_{0}} \int_{-a}^{a}|\Theta(x+i y)|^{p} d x<N_{p}
$$

if and only if there is a $Q_{p}$ such that

$$
\sup _{0<y \leq y_{0}} \int_{-a}^{a}\left|\Theta_{0}(x+i y)\right|^{p} \frac{d x}{x^{2}+(1+y)^{2}}<Q_{p} .
$$

Also, the hypothesis that $\Theta^{+}$is in $L^{p}$, for some $p>1$, implies that the function

$$
\Theta_{0}^{+}(x)=\exp \left[\frac{1}{\pi} \int \frac{\theta(t)}{t-x} \frac{1+t x}{1+t^{2}} d t+i \theta(x)\right]
$$

is in $L^{p}\left(d t /\left(t^{2}+1\right)\right)$. The function $\Theta_{0}^{+}$is the limit of $\Theta_{0}(x+i y)$ as $y \downarrow 0$.

We next transform our functions to the disc. For $|\zeta|<1$, we define $\Psi(\zeta)=$ $\Theta_{0}(i(1-\zeta) /(1+\zeta))$ and, for $-\pi<t<\pi$, we define $g\left(e^{i t}\right)=\theta(\tan t / 2)=$ $\theta\left(i\left(1-e^{i t}\right) /\left(1+e^{i t}\right)\right)$.

The conformal map $z=i(1-\zeta) /(1+\zeta)$ of $\pi_{+}$to the disc carries the lines $x=$ constant to circles orthogonal to the unit circle. It follows that

$$
\Psi\left(e^{i t}\right)=\operatorname{Lim}_{r \rightarrow 1} \Psi\left(r e^{i t}\right)=\exp \left[\tilde{g}\left(e^{i t}\right)+i g\left(e^{i t}\right)\right] ;
$$

here, we have set $\tilde{g}\left(e^{i t}\right)=(2 \pi)^{-1} \int_{-\pi}^{\pi} g\left(e^{i s}\right) c t((s-t) / 2) d s$. The function $\tilde{g}$ is called the conjugate of $g$. The hypothesis on $\Theta_{0}^{+}$implies that $\Psi\left(e^{i t}\right)$ is in $L^{p}$ on the unit circle.

From the representation

$$
\Psi(\zeta)=\exp \left[\frac{i}{2 \pi} \int_{-\pi}^{\pi} \frac{e^{i t}+\zeta}{e^{i t}-\zeta} g\left(e^{i t}\right) d t\right],
$$

it is clear that $\Psi\left(r e^{i t}\right)=\exp [-\tilde{g}(r, t)+i g(r, t)]$, where $g(r, t)$ is the harmonic function $\int_{-\pi}^{\pi}(2 \pi)^{-1} \operatorname{Re}\left[\left(e^{i s}+r e^{i t}\right) /\left(e^{i s}-r e^{i t}\right)\right] g\left(e^{i s}\right) d s$ and $\tilde{g}$ is its harmonic conjugate. It follows from a result of Zygmund [15, p. 164, Exercise 3] that there is an $A_{q}>0$ such that 


$$
\sup _{0 \leq r<1} \int_{-\pi}^{\pi} \exp \tilde{q}|g(r, t)| d t<A_{q},
$$

whenever $q<\pi / 2\left\|_{g}\right\|_{\infty}$; here, $\|g\|_{\infty}$ is the essential supremum of $g$ over the unit circle.

Therefore, $\Psi$ belongs to $H^{q}$ of the disc for some $q>0$ (in fact, any $q<$ $\pi / 2\|g\|_{\infty}$ will do). The remark that the boundary function of $\Psi$ belongs to $L^{p}$ implies that $\Psi$ belongs to $H^{p}$.

It is easy to finish the proof of Lemma 2. There is a $Q_{p}$ such that (11) holds if and only if there is an $S_{p}$ such that

$$
\sup _{0<y_{\leq} y_{0}} \int_{\Gamma_{y}}|\Psi(\zeta)|^{p} d|\zeta|<S_{p}
$$

The existence of $S_{p}$ follows from Lemma 1 and this completes the proof of Lemma 2.

3. Let $E$ be a measurable subset of reals and $\chi_{E}$ its characteristic function. The notation $m_{E}(y)$ will be used for the distribution of $\left|\pi \tilde{\chi}_{E}\right|$. Therefore, $m_{E}(y)=$ measure $\left\{t:\left|\tilde{\chi}_{E}(t)\right| \geq y\right\}$. Similarly, $\mu_{E}(y)$ is the distribution $\mu_{E}(y)=$ measure $\left\{t \in E:\left|\tilde{\chi}_{E}(t)\right| \geq y\right\}$.

The following lemma appears in Zygmund [14, p. 15].

Lemma 3. Let $E$ be a bounded measurable subset of reals, then, for $y \geq 0$, $m_{E}(y)=2|E| / \sinh y$ and $\mu_{E}(y)=2|E| /\left(e^{y}+1\right)$; here, $|E|$ denotes the measure of $E$.

We now introduce three functions which will be used to obtain generalized solutions of $T_{E} f=0$. We define

$$
\begin{aligned}
& \theta_{1}(t)=\left[\operatorname{tg}^{-1} t-(\pi / 2) \operatorname{sgn} t\right] \chi_{E}(t), \\
& \theta_{2}(t)=\left[\operatorname{tg}^{-1} t+\pi / 2\right] \chi_{E}(t), \text { and } \\
& \theta_{3}(t)=\left[\operatorname{tg}^{-1} t-\pi / 2\right] \chi_{E}(t) ;
\end{aligned}
$$

here, $-\pi / 2<t g^{-1} t<\pi / 2$. Obviously, if $E \subset[0, \infty)$ or $E \subset(-\infty, 0]$, then these three functions will not be distinct.

Our next theorem permits us to use the techniques of $\$ 2$ for the functions $\theta_{1}, \theta_{2}, \theta_{3}$.

Theorem 2. Let $\Theta_{i}(z)=\exp \left[\pi^{-1} \int \theta_{i}(t)(t-z)^{-1} d t\right]-1$, where $\theta_{i}$ are defined by (17), for $i=1,2,3$. Then $\Theta_{i} \in H_{+}^{p}$, for some $p>1$ and $i=1,2,3$. In fact, $\Theta_{1} \in H_{+}^{p}$, for any $p<2$. 
Proof. We have already noted in equation (4) that $\Theta_{i}^{+}=\exp \left[\tilde{\theta}_{i}+i \theta_{i}\right]-1$. Let $a, b$ be real numbers such that $b>a>1$ and $E \subset[-a, a]$.

For $|x|>b, \theta_{i}(x)=0$ and hence, $\left|\Theta_{i}^{+}(x)\right| \leq \exp \left|\tilde{\Theta}_{i}(x)\right|-1$.

Another easy power series argument can be used to establish that $\Theta_{i}^{+} \epsilon$ $L^{p}(R \sim[-b, b])$, for any $p>1$ and $i=1,2,3$.

It suffices to prove that the functions $\exp \tilde{\theta}_{i}$ are in $L^{p}(-b, b)$, for some $p>1$. Using the fact that $\left|\left(t g^{-1} x-t g^{-1} t\right)(x-t)^{-1}\right|$ is bounded, it follows that

$$
\exp \tilde{\theta}_{i} \leq A \exp \left|\theta_{i} \tilde{X}_{E}\right|, \quad i=1,2,3,
$$

where $A$ is some constant. The argument to obtain (18) when $i=1$ requires considering separately the cases where $x>0$ and $x<0$.

Clearly, for $i=1,2,3$, there is a $\gamma<1$ such that $\left|\pi^{-1} \theta_{i}(x)\right|<\gamma$ on $[-b, b]$; moreover, $\left|\pi^{-1} \theta_{1}(x)\right| \leq 1 / 2$. Therefore,

$$
\exp \tilde{\theta}_{i} \leq A \exp \gamma\left|\pi \tilde{\chi}_{E}\right|
$$

Now,

$$
\int_{-b}^{b} \exp p \gamma\left|\pi \tilde{\chi}_{E}\right| d t=-\int_{0}^{\infty} \exp p \gamma y d m_{b}(y)
$$

where $m_{b}(y)=$ measure $\left\{t \in[-b, b]:\left|\pi \tilde{\chi}_{E}(t)\right| \geq y\right\}$. Let $y_{0}>0$ be such that $\left|\pi \tilde{\chi}_{E}(t)\right|<y_{0}$, for $t \notin[-b, b]$. Then using Lemma 3 we obtain

$$
\begin{aligned}
-\int_{0}^{\infty} \exp p \gamma y d m_{b}(y) & =-\int_{0}^{y_{0}} \exp p \gamma y d m_{b}(y)-\int_{y_{0}}^{\infty} \exp p \gamma y d m_{E}(y) \\
& =-\int_{0}^{y_{0}} \exp p \gamma y d m_{b}(y)+4|E| \int_{y_{0}}^{\infty} \exp (p \gamma y) \frac{e^{y}+e^{-y}}{\left[e^{y}-e^{-y}\right]^{2}} d y .
\end{aligned}
$$

These last two integrals both converge if $p<1 / \gamma$. Observe that we have demonstrated that $\Theta^{+}$is in $L^{p}$ for any $p<2$. An application of Lemma 2 completes the proof.

When $\theta_{1}, \theta_{2}, \theta_{3}$ are used for $\theta$ in equation (6) we obtain $T_{E} f_{i}=\chi_{E}$, $i=1,2,3$, where, for $x \in E$,

$$
f_{1}(x)=\frac{\operatorname{sgn} x \exp \tilde{\theta}_{1}(x)}{\left(x^{2}+1\right)^{1 / 2}}, f_{2}(x)=-\frac{\exp \tilde{\theta}_{2}(x)}{\left(x^{2}+1\right)^{1 / 2}}, \quad \text { and } \quad f_{3}(x)=+\frac{\exp \tilde{\theta}_{3}(x)}{\left(x^{2}+1\right)^{1 / 2}} .
$$

The following theorem results:

Theorem 3. Let $\theta_{1}, \theta_{2}$ and $\theta_{3}$ be defined by (17), then the function $f_{2}-f_{3}$, where $f_{2}$ and $f_{3}$ are defined by (19), is a generalized eigenfunction of the equation $T_{E} f=0$. The function $f_{1}$, defined by (19), is an $L^{p}(E)$ solution of $T_{E} f=\chi_{E}$, for every $p<2$.

Proof. It is clear that both of the functions $f_{2}-f_{3}$ and $f_{1}$ are nonzero. It follows from Theorem 2 that $f_{2}-f_{3}$ belongs to $L^{p}(E)$, for some $p>1$, and $f_{1}$ 
belongs to $L^{p}(E)$ for any $p<2$. Alternatively, one can use the distribution $\mu_{E}$ to obtain these conclusions.

These may be further choices of the function $\theta$ which reduce equation (6) to the identity $T_{E} f=\chi_{E}$. The three functions $\theta_{1}, \theta_{2}$ and $\theta_{3}$ defined by (17) exhaust the author's list of functions where equation (6) reads $T_{E} f=\chi_{E}$ with $f \in L^{p}(E)$, for $p>1$. It is easy to make choices of $E$ for which the functions $f_{1}$, $f_{2}$ and $f_{3}$ are linearly independent. Also, if for example $E=[a, b]$, where $0 \in(a, b)$, then one observes that $f_{1}$ and $f_{2}$ are linearly independent and that $f_{1}-f_{2}$ is the unique nonzero $L^{2}(E)$ solution of $T_{E} f=0$. It is always true that the dimension of the solution space of $T_{E} f=0$ in $L^{2}(E)$ is less than or equal to 1. This follows from the fact that $T_{E}$ is completely seminormal and $T_{E}^{*} T_{E}-$ $T_{E} T_{E}^{*}$ is the one dimensional operator $-(2 \pi)^{-1}\left(, \chi_{E}\right) \chi_{E}$.

Further choices of $E$, for example $E=[-1,-1 / 2] \cup[-1 / 4,1 / 4] \cup[1 / 2,1]$, show that our technique sometimes fails to give the $L^{2}(E)$ solution of $T_{E} f=0$. The existence of this solution follows from the results on the essential spectra of $T_{E}$ in [2] or Putnam [9].

It is also obvious from our last example that the dimension of the solution space of $T_{E} f=0$ in $L^{p}(E)$, where $1<p<2$, may be greater than 1 .

4. Throughout this section it is assumed that

$$
\begin{aligned}
E=\left[a_{1}, b_{1}\right] \cup \cdots \cup\left[a_{n}, b_{n}\right] \cup \cdots \cup[0,1] & ; \\
& \text { here }-1=a_{1}<b_{1}<a_{2}<b_{2}<\cdots<0 \text { and } a_{n} \rightarrow 0 .
\end{aligned}
$$

The functions $f_{1}, f_{2}$ will continue to be the functions defined in (19). It follows from the previous section that $g=f_{1}-f_{2}$ is a generalized eigenfunction of $T_{E} f=0$. In fact, with very little work, one can show that $g$ is in $L^{p}(E)$, for $p<2$. In this section we obtain a set of conditions on $E$ sufficient for $g$ to belong to $L^{2}(E)$.

Set $E^{-}=E \cap[-1,0], E^{+}=E \cap(0,1]$ and $E_{p}=\left[a_{1}, b_{1}\right] \cup \ldots \cup\left[a_{p}, b_{p}\right]$. It follows that

$$
\exp \tilde{\chi}_{E^{+}}(x)=|x|^{-1}|1-x| \text { and } \exp \pi \tilde{\chi}_{E_{p}}(x)=\prod_{k=1}^{p} \frac{\left|x-b_{k}\right|}{\left|x-a_{k}\right|} .
$$

Computations similar to those performed in $\S_{3}$ show that $f_{1}$ and $f_{2}$ are in $L^{2}(E)$ if $g_{1}=\exp \left(y^{-} \pi \tilde{\chi}_{E^{+}}\right) \cdot \exp \left(y^{+} \pi \tilde{\chi}_{E^{-}}\right)$and $g_{2}=\exp \left(y^{+} \pi \tilde{\chi}_{E}\right)$ belong to $L^{1}(E)$, respectively; here, we have set $y^{ \pm}=2\left(\pi^{-1}\right) \operatorname{tg}^{-1}(x) \pm 1$.

Lemma 4. The function $g_{1}$ is in $L^{1}(0,1)$. If $g_{2}$ is in $L^{1}\left(E^{-}\right)$, then $g_{1}$ is in $L^{1}\left(E^{-}\right)$. 
Proof. For $x>0, \exp \left(y^{+}(x) \pi \tilde{X}_{E}-(x)\right) \leq 1$. Therefore, $g_{1}(x) \leq(x /(1-x))\left|y^{-}\right|$ and since $\left|y^{-}(1)\right|=1 / 2$, it follows that $g_{1}$ is in $L^{1}(0,1)$. For $x<0, g_{1}(x) \leq g_{2}(x)$ and this observation completes the proof of the lemma.

The task of finding conditions sufficient for $g_{2}$ to be in $L^{1}(E)$ is more difficult.

For $x>0, \exp \left(\left(\operatorname{tg}^{-1} x \tilde{\chi}_{E}-(x)\right)\right) \leq 1$. This observation along with the observation that $\exp \left(\operatorname{tg}^{-1} x \tilde{\chi}_{E^{+}}(x)\right)=\left\{x^{-1}(1-x)\right\}^{\pi^{-1}} t^{-1} x$ is bounded on $(0,1)$ implies that $g_{2}$ is in $L^{1}(0,1)$ if $\exp \pi \tilde{\chi}_{E}$ is in $L^{1}(0,1)$.

Our next lemma describes a class of sets of the form (20) for which exp $\pi \widetilde{\chi}_{E}$ belongs to $L^{1}(0,1)$. Clearly, the question of when $\exp \pi \tilde{X}_{E}$ is in $L^{1}(0,1)$ depends only on $a_{n}$ and $b_{n}$ for large $n$. Moreover, if $E$ and $F$ are two sets of the form (20) with $E^{-} \subset F^{-}$, then, when $\exp \pi \tilde{\chi}_{E}$ is in $L^{1}(0,1)$, it follows that $\exp \pi \tilde{\chi}_{F}$ is in $L^{1}(0,1)$.

Lemma 5. Fix $a>\sqrt{2}$. Suppose, that for $n \geq N_{0}, a_{n}=-a^{-a^{n}}$ and $b_{n}=a^{-n} a_{n}$. Then $\exp \pi \tilde{X}_{E}$ is in $L^{1}(0,1)$.

Proof. For $x>0$, let $k_{x}=\min \left\{k:\left|b_{k}\right| \leq x\right\}$. Then

$$
\begin{aligned}
\exp \pi \tilde{\chi}_{E}(x) & \leq C x^{-1} \exp \pi \tilde{\chi}_{E_{k_{x}}}(x) \leq C x^{-1} \prod_{j=N_{0}}^{k_{x}} \frac{x+\left|b_{j}\right|}{x+\left|a_{j}\right|} \\
& \leq C x^{-1} \prod_{j=N_{0}}^{k_{x}} 2 \frac{\left|b_{j}\right|}{\left|a_{j}\right|} \leq C^{\prime \prime} x^{-1} 2^{k_{x}} a^{-k_{x}\left(k_{x}-1\right) / 2}
\end{aligned}
$$

here, $C, C^{\prime \prime}$ are constants. For $x$ small, $k_{x}$ is large and we can assume $\left(k_{x}-1\right) / 2>4$. Using the fact that $a>\sqrt{2}^{x}$ we obtain, for small $x$,

$$
\exp \pi \tilde{\chi}_{E}(x) \leq C^{\prime \prime} x^{-1} a^{-2 k_{x}} \text {. }
$$

The fact that $\left|b_{k_{x}}\right|=a^{-k_{x}}\left|a_{k_{x}}\right| \leq x$ implies that $a^{k_{x}} \geq-1 / 2 \log _{a} x$. Therefore, for $x$ small, $\exp \pi \widetilde{X}_{E}(x) \leq 4 C^{\prime \prime} x^{-1}\left(\log _{a} x\right)^{-2}$ and this completes the proof.

Finally, we give conditions sufficient for $g_{2}$ to belong to $L^{1}\left(E^{-}\right)$.

Lemma 6. Let $E$ be a set of the form (20), then the conditions

$$
\begin{array}{r}
\sum_{p=N_{0}}^{\infty} \frac{\left|a_{p}-a_{p+1}\right|}{\left|a_{p-1}-a_{p}\right|}<\infty, \\
\sum_{p=N_{0}}^{\infty} \frac{\left|b_{p-1}-a_{p}\right|^{1 / 2}}{\left|a_{p-1}-a_{p}\right|\left|a_{p+1}\right|}<\infty
\end{array}
$$


are sufficient to imply that $g_{2}$ is in $L^{1}\left(E^{-}\right)$; bere, $N_{0} \geq 2$.

Proof. The following estimate holds for $a_{p}<x<b_{p}$ :

$$
\begin{aligned}
g_{2}(x) & \leq \exp \left(y+\pi \tilde{\chi}_{E_{p-1}}\right) \cdot \exp \left(y+\tilde{\chi}_{\left[a_{p}, 0\right]}\right) \cdot\left\{\frac{|1-x|}{|x|}\right\}^{y+} \leq C \frac{\exp \left(y+\pi \tilde{\chi}_{E_{p}}\right)}{\left[b_{p}-x\right]^{y^{+}}} \\
& \leq C\left\{\frac{x-b_{p-1}}{x-a_{p}}\right\}^{y^{+}} \frac{1}{\left(x-a_{p-1}\right)^{y}} \leq C \frac{1}{\left[a_{p}-a_{p-1}\right]^{y}}{ }^{\mp\left(b_{p}\right)}\left\{1+\frac{a_{p}-b_{p-1}}{x-a_{p}}\right\}^{y^{+}\left(b_{p}\right)},
\end{aligned}
$$

where $C$ is a positive constant.

Using the inequality $(1+t)^{a} \leq 1+t^{a}$, for $t \geq 1$ and $0<a<1$, we obtain, for $a_{p}<x \leq a_{p}+\left(a_{p}-b_{p-1}\right)$,

$$
g_{2}(x) \leq \frac{C}{\left(a_{p}-a_{p-1}\right)^{y^{+}\left(b_{p}\right)}}\left[1+\left\{\frac{a_{p}-b_{p-1}}{x-a_{p}}\right\}^{y^{+}\left(b_{p}\right)}\right]
$$

and, for $a_{p}+\left(a_{p}-b_{p-1}\right)<x<b_{p}$,

$$
g_{2}(x) \leq \frac{2 C}{\left(a_{p}-a_{p-1}\right)^{y^{+}\left(b_{p}\right)}} .
$$

In these last estimates if $a_{p}+\left(a_{p}-b_{p-1}\right) \geq b_{p}$, then the estimate (23) is used for all $x$ in $\left(a_{p}, b_{p}\right)$.

It now follows by direct integration from (23) and (24) and use of the fact that $\left|b_{p}\right|^{-1} g^{-1}\left|b_{p}\right| \rightarrow 1$ that

$$
\int_{a_{p}}^{b_{p}} g_{2}(x) d x \leq C^{\prime \prime}\left[\frac{a_{p+1}-a_{p}}{a_{p}-a_{p-1}}+\frac{\left(a_{p}-b_{p-1}\right)^{y^{+}\left(b_{p}\right)}}{\left(a_{p}-a_{p-1}\right)\left|b_{p}\right|}\right],
$$

where $C^{\prime \prime}$ is a positive constant.

The conditions (21) and (22) follow easily from (25).

The result of Lemmas 4, 5, and 6 is the following theorem.

Theorem. There exist sets $E$ of the form (20) with nonzero $L^{2}(E)$ solutions of $T_{E} f=0$. The point $\lambda=0$ is a boundary eigenvalue of $T_{E}$ on $L^{2}(E)$.

Proof. If $a>\sqrt{2}$ and, for $n \geq N_{0}, a_{n}=-a^{-a^{n}}$, then (21) converges. If now, for $n \geq N_{0}+1, b_{n-1}$ is chosen such that $a_{n-1}<a^{-(n-1)} a_{n-1}<b_{n-1}<a_{n}$ and close enough to $a_{n}$ so that (22) converges, then $g=f_{1}-f_{2}$ defined by (19) is a nonzero $L^{2}(E)$ solution of $T_{E} f=0$. The fact that $\lambda=0$ is a boundary point of the spectrum of $T_{E}$ follows from [3].

Added in proof (July 1973): In a recent announcement R. W. Carey and J. D. Pincus, On an invariant for certain operator algebras, obtain necessary and sufficient conditions for zero to be an eigenvalue of the operator defined in equation (1). 


\section{REFERENCES}

1. J. E. Brennan, Invariant subspaces for a class of subnormal operators (preprint).

2. K. F. Clancey, The essential spectrum of some singular integral operators, Amer. J. Math. (to appear).

3. - Seminormal operators with compact self-commutator, Proc. Amer. Math. Soc. 26 (1970), 447-454. MR $42 \# 885$.

4. P. Duren, Theory of $H^{p}$ spaces, Pure and Appl. Math., vol. 38, Academic Press, New York, 1970. MR $42 \# 3552$.

5. R. M. Gabriel, An inequality concerning the integrals of positive subharmonic functions along certain circles, J. London Math. Soc. 5 (1930), 129-131.

6. E. Hille and J. D. Tamarkin, On the absolute integrability of Fourier transforms, Fund. Math. 25 (1935), 329-352.

7. J. D. Pincus, Commutators and systems of singular integral equations. I, Acta Math. 121 (1968), 219-249. MR 39 \# 2026.

8. C. R. Putnam, Commutation properties of Hilbert space operators and related topics, Ergebnisse der Mathematik und ihrer Grenzgebiete, Band 36, Springer-Verlag, New York, 1967. MR 36 \# 707.

9. - The spectra of semi-normal singular integral operators, Canad. J. Math. 22 (1970), 134-150. MR 41 \#315.

10. - Eigenvalues and boundary spectra, Illinois J. Math. 12 (1968), 278-282. MR 37 \# 2030.

11. J. G. Stampfli, A local spectral theory for operators. III: Resolvents, spectral sets and similarity (preprint).

12. F. Tricomi, Integral equations, Interscience, New York, 1957. MR 20 \#1177.

13. H. Widom, Singular integral equations in $L^{p}$, Trans. Amer. Math. Soc. 97 (1960), 131-160. MR $22 \# 9830$.

14. A. Zy gmund, Intégrales singulières, Lecture Notes in Math., vol. 204, SpringerVerlag, New York, 1971.

15. — , Trigonometrical series, 2nd. rev. ed., Cambridge Univ. Press, New York, 1959. MR $21 \# 6498$.

DEPARTMENT OF MATHEMATICS, UNIVERSITY OF GEORGIA, ATHENS, GEORGIA 30602 